\title{
A 3D Procrustean Approach to Transform WGS84 Coordinates to Ghana War Office 1926 Reference Datum*
}

\author{
${ }^{1}$ B. Kumi-Boateng, ${ }^{1}$ Y.Y. Ziggah \\ ${ }^{1}$ University of Mine and Technology, P. O. Box 237, Tarkwa
}

Kumi-Boateng, B. and Ziggah, Y.Y. (2020) "A 3D Procrustean Approach to Transform WGS84 Coordinates to Ghana War Office 1926 Datum”, Ghana Mining Journal, Vol. 20, No. 1, pp. 1 - 10.

\begin{abstract}
Satellite positioning technique such as Global Positioning System (GPS) is available to all countries for geospatial positioning. The availability of such positioning technique has revolutionised surveying in Ghana. The GPS operates on a global reference frame to fix control points for surveying and mapping purposes. There is therefore the need to transform coordinates from the satellite-based datum to the Ghana War Office 1926 datum. Several iterative methods have been proposed over the years for coordinate transformation and have been found to exhibit good transformation accuracy. However, these iterative methods always demand the linearisation of the transformation model equations and initial approximation values of the yet to be determined transformation parameters. These computational processes further enhance the computational complexity of the iterative methods and longer convergence time. As alternative solution, the Procrustes method has been proposed and applied to solve coordinate transformation problems in different geodetic reference networks. Review of previous studies indicates that the Procrustes method is direct, simple to use and produce satisfactory transformation accuracy. This method, however, is yet to be applied to ascertain its efficiency in the Ghana geodetic reference network. Therefore, this study utilised the 3D Procrustean approach to transform coordinates from World Geodetic System 1984 (WGS84) to Ghana War Office 1926 reference datum. The technique produced Root Mean Square Horizontal Error (RMSHE), Arithmetic Mean of the Horizontal Error (AMHE) and Standard Deviation (SD) values of $1.003 \mathrm{~m}, 0.901$ $\mathrm{m}$ and $0.452 \mathrm{~m}$, respectively. This study is serving as an extension to the ongoing research works to determine optimal transformation model for Ghana geodetic reference network.
\end{abstract}

Keywords: Procrustean Approach, Coordinate Transformation, Conformal Model, Satellite Positioning

\section{Introduction}

Survey controls position fixing using satellite based positioning techniques, such as GPS which relies on WGS84 as reference datum, has been on the rise in Ghana over the past two decades. Because the GPS reference datum (WGS84) is globally established, it has become necessary to transform coordinates from the WGS84 into Ghana local geodetic system (War Office 1926) and vice versa (Ayer and Fosu, 2008; Poku-Gyamfi, 2009; Ziggah et al., 2019). This would help to establish a transformation model that can appropriately fits the WGS84 data into Ghana's geodetic geometry for surveying and mapping applications.

Generally, the traditional approach is to perform seven parameter datum transformation by linearising a set of nonlinear numerical equations and then applying the least squares method to iteratively determine the transformation parameters (Grafarend and Awange, 2003; Awange and Grafarend, 2005; Awange and Paláncz, 2016). These iterative transformation procedures require initial approximation values of the yet to be determined transformation parameters and this takes longer period to reach the convergence criteria. These issues of computational complexity and uncertainties in the initial starting values have led to the widespread formulation of several analytical methods that are direct, simple to use and offer fast processing capabilities (Păun et al., 2017).

One of the widely used analytical methods for solving the seven parameter transformation problem is the Procrustes approach (Grafarend and Awange, 2003; Paláncz et al., 2010). With this technique, the transformation parameters can be determined directly by only inserting the source and target datum coordinates into the Procrustes algorithm (Awange and Paláncz, 2016; Awange and Grafarend, 2005). In addition, the Procrustes approach is computationally efficient and avoids linearization of nonlinear numerical transformation equations.

In the literature, the efficiency of Procrustes approach has been well documented; but the method is unexplored in the Ghana geodetic reference network. It is necessary, at this stage, to clarify the need to apply the Procrustes method in Ghana. The following are some of the reasons:

(i). In Ghana, coordinate transformation research is usually done between the Ghana War Office 1926 and WGS84. The War Office 1926 ellipsoid presently in use which has the Accra 1929 datum as its reference frame is an astro-geodetic datum. This implies that there is a need to apply a more pragmatic approach capable of adequately harmonising data related to the 
global datum (WGS84) and War Office 1926 datum.

(ii). The local geodetic network of Ghana has been recognised to be highly distorted due to multitude of problems generally associated with the establishment of local geodetic network and quality of data associated with it (Poku-Gyamfi, 2009; Ayer, 2008; Ayer and Fosu, 2008). The intention, therefore, is to apply a transformation model that has the ability to adequately account for the heterogeneity existing in the spatial data related to the War Office 1926 datum.

In view of these enumerated issues, it has become practical to explore and apply other consistent coordinate transformation method in Ghana geodetic reference network. Therefore, this study introduces the implementation of the Procrustean approach to perform coordinate transformation between WGS84 ellipsoidal datum and Ghana War Office 1926 ellipsoid. The study is a contribution towards the ongoing quantitative evaluation of different transformation methods with the aim of selecting the optimal approach for Ghana.

\section{Resources and Methods Used}

\subsection{Study Area and Data Description}

The study area is Ghana situated in West Africa. It covers a maximum of $672 \mathrm{~km}$ from North to South between latitudes $4^{0} 30^{\prime} \mathrm{N}$ and $11^{\circ} \mathrm{N}$, and $536 \mathrm{~km}$ East to West between longitudes $3^{0} \mathrm{~W}$ and $1^{0} \mathrm{E}$ (Mugnier, 2000). Historical information shows that Ghana geodetic survey began in June 1904 by Captain F.G. Gordon Guggisberg of the by then Governor of the Gold Coast Colony now Ghana. The Governor made observations for latitude from a pillar located in the compound of the house of the Secretary of Native affairs in Accra with a zenith telescope to fifteen stars that gave a final probable error of the azimuth at 0.360" (Poku-Gyamfi, 2009). It has been reported that traversing was carried out to connect that pillar to a Gold Coast Survey (G.C.S) beacon No. 547 in Accra. The longitude of G.C.S 547 was determined the same year between the months of November and December through the exchange of telegraphic signals with Cape Town, South Africa (Mugnier, 2000).

The G.C.S 547 (Accra) had latitude $5^{0} 23^{\prime} 43.33^{\prime \prime} \mathrm{N}$ and longitude $0^{\circ} 11^{\prime} 52.3^{\prime \prime} \mathrm{W}$ and was adopted as the basic latitude and longitude for the then Gold Coast Colony although the influence of local attraction was noticed in the observations after later works. Astronomical observations were further carried out and the result was comparable to the trigonometric coordinates. These formed the basis for the computation of geographical coordinates and adoption of the War Office 1926 ellipsoid as the best locally fit ellipsoid for the then Gold Coast Colony. The British War Office computed ellipsoid parameters for the War Office 1926 ellipsoid are the semi minor axis $b=6356751.68824042 \mathrm{~m}$, semi-major axis $a=6378299.99899832 \mathrm{~m}$ and flattening $f=1 / 296$ (Poku-Gyamfi, 2009; Ayer, 2008; Ayer and Fosu, 2008).

The terrestrial reference frame realisation was done using trigonometric stations that led to the establishment of the Accra 1929 datum using the fundamental point of station G.C.S 547 and adopted astronomical azimuth on the War Office 1926 ellipsoid. Later, it was realised that for the purposes of surveying and mapping, there was the need to have a more convenient system other than the geographic coordinates. Consequently, the then Gold Coast now Ghana adopted a plane rectangular coordinate system on the Transverse Mercator (TM) Projection (Mugnier, 2000; Poku-Gyamfi, 2009). The Transverse Mercator started on longitude $01^{0} 00^{\prime} \mathrm{W}$ (central meridian) and latitude $04^{0} 40^{\prime} \mathrm{N}$. In order to avoid negative coordinates, False Easting value of $274319.736 \mathrm{~m}$ was added to all Y coordinates with False Northing set to zero. A scale factor of 0.99975 was found to be more appropriate at the central meridian. The Easting and Northing projected grid coordinates based on the Transverse Mercator (TM) $1^{0} \mathrm{NW}$ is the positional information found on survey maps in Ghana.

In pursuit of enhancing the use of Global Navigation Satellite System (GNSS) to meet the growing demand for land related positioning activities, led to the redefining of the Ghana geodetic reference network (Wonnacot, 2007; Poku-Gyamfi and Schueler, 2008). Moreover, the goal for African countries to have a common reference frame under the African Geodetic Reference Frame (AFREF) based on the International Terrestrial Reference System (ITRS) also contributed to the redefinition of Ghana geodetic network.

In view of the above, a nationwide project to establish GNSS reference station network was embarked on by the Ghana Survey and Mapping Division of Lands Commission through the Land Administration Project (LAP) funded by the World Bank. This project was divided into phases, with the completed first phase referred to as the Golden triangle (Fig. 1). The Golden Triangle is made up of three permanent stations having $100 \mathrm{~km}$ radius of coverage situated at the vertices of the three largest cities (Sekondi-Takoradi, Kumasi and Accra) where almost all the natural resources like 
gold, oil, timber, cocoa, diamond, manganese, bauxite among others are located in Ghana (PokuGyamfi, 2009). These three permanent reference stations form a triangle of sides $200 \mathrm{~km}$ that cover a total area of $79857 \mathrm{~km}^{2}$ representing $33.5 \%$ of the total land mass $\left(238540 \mathrm{~km}^{2}\right)$ of Ghana (Fosu et al., 2006).

In this GNSS reference station network, a continuous twelve-hour observation was made on 19 historical triangulation stations located in the Golden Triangle by dual frequency GPS receivers. The coordinates provided by the GPS receivers were then differentially processed with the International GNSS permanent stations (IGS) to obtain the respective common points on the WGS84 ellipsoid. These 19 GNSS-derived coordinates are based on the International Terrestrial Reference Frame 2005 (ITRF05) specified at epoch 2007.39 (Kotzev, 2013).

Table 1 shows the Cartesian coordinates of common points applied in this study to carry out the coordinate transformation. These coordinates are found in the Golden Triangle. Also, the Ghana Survey and Mapping Division provided legacy projected grid coordinates related to the Accra 1929 datum to help evaluate the performance of the Procrustes approach.

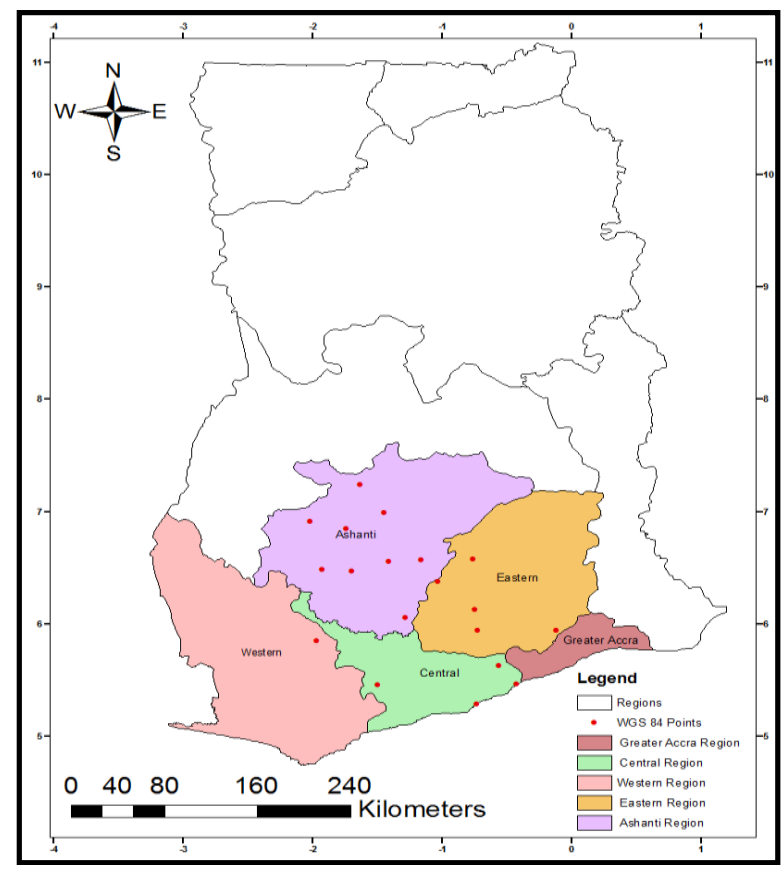

Fig. 1 Study Area: Regions Covered by the Golden Triangle Points

Table 1 Data Used for the Procrustes Coordinate Transformation (Units in metres)

\begin{tabular}{|c|c|c|c|c|c|c|}
\hline PT ID & War X & War Y & War Z & WGS X & WGS Y & WGS Z \\
\hline 1 & 6349409.327 & -46970.670 & 602528.309 & 6349216.526 & -46937.595 & 602850.422 \\
\hline 2 & 6347511.181 & -61996.956 & 620767.330 & 6347317.555 & -61963.994 & 621089.479 \\
\hline 3 & 6347456.143 & -166364.103 & 601974.614 & 6347263.655 & -166331.080 & 602299.601 \\
\hline 4 & 6350895.804 & -81439.118 & 583003.237 & 6350704.167 & -81406.532 & 583324.885 \\
\hline 5 & 6344311.354 & -13541.146 & 655229.232 & 6344115.678 & -13508.014 & 655552.515 \\
\hline 6 & 6338161.167 & -114348.252 & 702846.772 & 6337963.682 & -114315.111 & 703169.740 \\
\hline 7 & 6341401.872 & -142407.686 & 667850.435 & 6341206.186 & -142375.250 & 668173.703 \\
\hline 8 & 6343764.185 & -80827.204 & 655678.421 & 6343568.942 & -80794.501 & 656000.509 \\
\hline 9 & 6336087.817 & -84696.251 & 725228.756 & 6335889.215 & -84663.620 & 725550.613 \\
\hline 10 & 6341585.798 & -82924.611 & 676032.731 & 6341389.492 & -82892.051 & 676355.510 \\
\hline 11 & 6335400.386 & -128828.430 & 724740.654 & 6335201.992 & -128794.655 & 725062.881 \\
\hline 12 & 6334220.039 & -212939.599 & 715227.300 & 6334022.452 & -212905.291 & 715549.999 \\
\hline 13 & 6325317.473 & -180075.852 & 797697.362 & 6325116.025 & -180042.328 & 798017.357 \\
\hline 14 & 6328359.648 & -222871.878 & 762205.871 & 6328160.051 & -222837.357 & 762526.598 \\
\hline 15 & 6329201.162 & -159721.648 & 770957.863 & 6329000.768 & -159688.632 & 771278.987 \\
\hline 16 & 6330226.210 & -192706.232 & 754944.210 & 6330026.793 & -192671.938 & 755265.209 \\
\hline 17 & 6334967.992 & -156168.638 & 723153.737 & 6334769.765 & -156134.602 & 723476.351 \\
\hline 18 & 6335189.568 & -187480.113 & 713775.381 & 6334991.985 & -187446.158 & 714097.595 \\
\hline 19 & 6341604.179 & -217730.715 & 645392.842 & 6341409.929 & -217696.318 & 645716.644 \\
\hline
\end{tabular}




\subsection{Methods Used}

A summary of the workflow of the Procrustes approach is shown in Fig. 2. Forward conversion was first conducted to obtain the respective Cartesian coordinates for both reference datums. The Procrustes algorithm was then applied to determine the transformation parameters. With the obtained parameters, the WGS84 Cartesian coordinates were transformed into the War Office
1926 datum. Reverse conversion was then employed on the transformed WGS84 Cartesian coordinates to obtain geodetic coordinates based on the War Office 1926 datum. Subsequently, these geodetic coordinates were then projected onto the Transverse Mercator to obtain the grid coordinates (Easting, Northing). Detailed mathematical presentation of the procedures is provided in the subsequent sections.

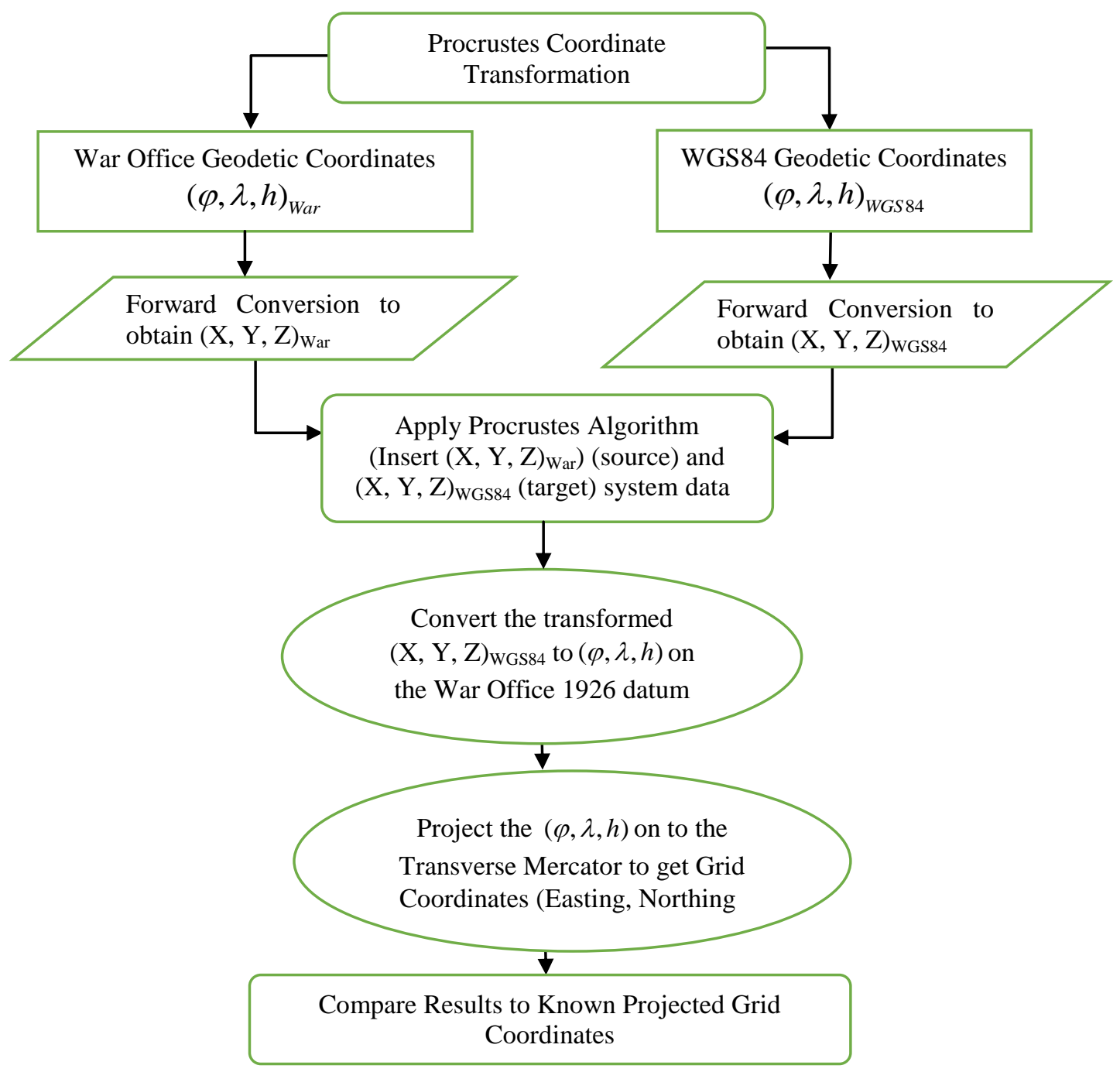

Fig. 2 Flow Chart of Procrustes Transformation Approach 


\subsubsection{Data Conversion}

The standard forward equation (Equation (1)) as given in Hofmann-Wellenhof and Moritz (2006) was used to convert both the War Office 1926 and WGS84 geodetic coordinates $(\varphi, \lambda, h)$ to Cartesian coordinates $(\mathrm{X}, \mathrm{Y}, \mathrm{Z})$.

$$
\begin{aligned}
& X=(Q+h) \cos \varphi \cos \lambda \\
& Y=(Q+h) \cos \varphi \sin \lambda \\
& Z=\left[Q\left(1-e^{2}\right)+h\right] \sin \varphi
\end{aligned}
$$

where the radius of curvature in the prime vertical (Q) is defined by Equation (2). $\varphi, \lambda$ and $h$ are the geodetic latitude, geodetic longitude and ellipsoid height, respectively.

$$
Q=\frac{a}{\sqrt{1-e^{2} \sin ^{2} \varphi}}
$$

where $a$ and $e$ are the semi-major axis and first eccentricity of the ellipsoid.

In the case of the reverse conversion, Bowring inverse formula (Bowring, 1976) as provided in Equations (3)-(5) were used.

$$
\begin{aligned}
& \phi=\arctan \left[\frac{Z+\varepsilon^{2} b \sin ^{3} q}{p-e^{2} a \cos ^{3} q}\right] \\
& \lambda=\arctan \left[\frac{Y}{X}\right] \\
& h=P \cos \varphi+Z \sin \varphi-a \sqrt{1-e^{2} \sin ^{2} \varphi}
\end{aligned}
$$

where $q$ is the parametric latitude (Equation (6)), $b$ is the semi-minor axis of the ellipsoid, $P$ is the perpendicular distance from the rotational axis (Equation (7)) and $\varepsilon$ (Equation (8)) is the second eccentricity.

$$
\begin{aligned}
& q=\arctan \left[\frac{a Z}{b P}\right] \\
& P=\sqrt{X^{2}+Y^{2}} \\
& \varepsilon=\frac{e^{2}}{1-e^{2}}
\end{aligned}
$$

\subsubsection{Procrustean Transformation Solution}

Procrustes is a minimisation approach that falls under the purview of multidimensional scaling techniques. The Procrustes approach has the ability to perform one-to-one correspondence between data patterns having identical dimensionality and quantity of points. The technique finds isotropic dilatation, reflection, rigid translation and rotation to successfully achieve the best match from one configuration to another (Awange and Grafarend,
2005; Awange and Paláncz, 2016 and references therein).

In adjustment terms, let $\{a, b, c\}$ and $\{x, y, z\}$ be the given coordinates in two different datums (Paláncz et al., 2010). The Procrustean solution to the seven parameter transformation problem with $N$ points is expressed in Equation (9) as

$$
\begin{aligned}
& {\left[\begin{array}{ccc}
a_{1} & b_{1} & c_{1} \\
\vdots & \vdots & \vdots \\
a_{N} & b_{N} & c_{N}
\end{array}\right]=\left[\begin{array}{ccc}
x_{1} & y_{1} & z_{1} \\
\vdots & \vdots & \vdots \\
x_{N} & y_{N} & z_{N}
\end{array}\right] R^{T} \Delta s+} \\
& {\left[\begin{array}{c}
1 \\
\vdots \\
1
\end{array}\right]\left[\begin{array}{lll}
x_{0} & y_{0} & z_{0}
\end{array}\right]+\mathrm{Er}}
\end{aligned}
$$

where $\Delta s$ is an unknown scalar value for the scale factor, $\mathrm{R} \in \mathbb{R}^{3 \times 3}$ is the unknown orthonormal matrix for the rotation matrix and $\mathrm{Er}=e_{i, j}$ is the error matrix for $i=1, \ldots N$ and $j=1, \ldots$. 3. In compact form, Equation (9) is given in Equation (10) as

$$
a b c=x y z \times R^{T} \times \Delta s+1 \times x y z_{0}^{T}+\mathrm{Er}
$$

where the scale factor $(\Delta s)$ corresponds to $s \in \mathbb{R}$.

The translation vector $\left(x_{0}, y_{0}, z_{0}\right)$ corresponds to $\{x, y, z\}_{0} \in \mathbb{R}^{3}$, and the rotation matrix is $R \in \mathbb{R}^{3 \times 3}$

Introducing a positive definite weight matrix (W) as given in Equation (11) as

$$
W=\operatorname{diag}\left(w_{1}, \ldots, w_{N}\right)
$$

The square of the Frobenius norm of the weighted Er gives Equation (12).

$$
\|\mathrm{Er}\|_{w}^{2}=\operatorname{tr}\left(E r^{T} W E r\right)
$$

Equation (12) poses a constrained optimisation problem which requires $E r$ to be minimised (Equation (13)) subject to the constraint given in Equation (14) (Paláncz et al., 2010).

$$
\begin{aligned}
& \left\|\operatorname{Er}\left(\Delta s, x y z_{0}, R\right)\right\|_{w}^{2} \rightarrow \min \\
& R^{T} R=I_{3} \text { and }\|R\|=+1
\end{aligned}
$$

Gower and Dijksterhuis (2004) provided detailed methodology for solving Equations (13) and (14), respectively. In this study, a summary of the procedure is provided as follows:

i. Calculate the centering matrix $D$ using Equation (15).

$$
D=I_{N}-\frac{1}{N} \times 1 \times 1^{T}
$$

where $I_{N}$ is the $N$ dimensional unit matrix.

ii. Calculate the $B$ matrix using Equation (16).

$$
B=a b c^{T} \times D^{T} \times W \times D \times x y z
$$

iii. Apply the Singular Value Decomposition (SVD) technique to the $B$ matrix as shown in Equation (17) to obtain the matrices $U, V$ and diag $\left(\beta_{1}, \beta_{2}, \beta_{3}\right)$. 


$$
B=U \times \operatorname{diag}\left(\beta_{1}, \beta_{2}, \beta_{3}\right) \times V^{T}
$$

iv. Compute the rotation matrix $(R)$ (Equation (18)) using the $U$ and $V$ obtained from the SVD (Equation (17)).

$$
R=U \times V^{T}
$$

v. The rotation matrix is then expressed as a skew matrix as given in Equation (19).

$$
\Xi=\left(R-I_{3}\right)\left(R+I_{3}\right)
$$

The parameters $p, q$ and $r$ can be computed using Equation (20).

$$
\Xi=\left[\begin{array}{ccc}
0 & -r & q \\
r & 0 & -p \\
-q & p & 0
\end{array}\right]
$$

vi. The scale parameter (Equation (21)) can then be expressed as:

$$
\Delta s=\frac{\operatorname{tr}\left(a b c^{T} \times D^{T} \times W \times D \times x y z \times R^{T}\right)}{\operatorname{tr}\left(x y z^{T} \times D^{T} \times W \times D \times x y z\right)}
$$

where $\{\operatorname{tr}\}$ is the trace of a matrix.

vii. Since the translation vector satisfies the normal equation (Equation (22)), Equation (23) is finally obtained.

$$
\begin{aligned}
& 1^{T} \times W \times 1 \times x y z_{0}=\left(a b c-x y z \times R^{T} \Delta s\right)^{T} \\
& \times W \times 1 \\
& x y z_{0}=\left(1^{T} W \times 1\right)^{-1}\left(a b c-x y z \times R^{T} \Delta s\right)^{T} \\
& \times W \times 1
\end{aligned}
$$

In this study, the Procrustes algorithm has been implemented in MATLAB.

\subsubsection{Transverse Mercator Projection}

The projected grid coordinate system used in Ghana is based on the Transverse Mercator. The formula for the Transverse Mercator projection is given in two folds as: forward mapping from ellipsoid to the plane and the reverse mapping from plane to ellipsoid. The formula for the forward mapping from ellipsoid to the Plane is given in Equations (24) and (25) for the Easting and Northing coordinates (Dzidefo, 2011).

$$
\begin{aligned}
& \frac{E}{k_{o} v}=\lambda \cos \varphi+\frac{\lambda^{3} \cos ^{3} \varphi}{6}\left(1-t^{2}+\eta^{3}\right)+ \\
& \frac{\lambda^{5} \cos ^{5} \varphi}{120}\left(5-18 t^{2}+t^{4} 14 \eta^{2}-58 t^{2} \eta^{2}\right) \\
& \frac{N}{k_{o} v}=\frac{S}{v}+\frac{\lambda^{2}}{2} \sin \varphi \cos \varphi+\frac{\lambda^{2}}{24} \cos ^{3} \varphi\left(5-t^{2}+\right. \\
& \left.9 \eta^{2}+\eta^{4}\right)+\frac{\eta^{6}}{720} \sin \varphi \cos ^{5} \varphi\left(61-58 t^{2}+t^{4}+\right. \\
& \left.270 \eta^{2}-330 t^{2} \eta^{2}\right)
\end{aligned}
$$

The equation for the reverse mapping from plane to ellipsoid for latitude and longitude is given in Equations (26) and (27) (Dzidefo, 2011).

$$
\begin{aligned}
& \varphi=\varphi f-\frac{1}{2}\left(1+\eta^{2}\right)\left[\frac{E}{k_{o} v}\right]^{2}+\frac{t}{24}\left(5+5 t^{2}+6 \eta^{2}\right. \\
& \left.-6 \eta^{2} t^{2}-3 \eta^{4}-9 t^{2} \eta^{4}\right)\left[\frac{E}{k_{o} v}\right]^{4}-\frac{t}{720}\left(61+90 t^{2}\right. \\
& \left.+45 t^{4}+107 \eta^{2}-162 t^{2} \eta^{2}-45 t^{4} \eta^{2}\right)\left[\frac{E}{k_{o} v}\right]^{6} \\
& \lambda=\sec \varphi f\left(\left[\frac{E}{k_{o} v}\right]-\frac{1}{6}\left[\frac{E}{k_{o} v}\right]^{3}\right)\left(1+2 t^{2}+\eta^{2}\right)+ \\
& \frac{1}{120}\left[\frac{E}{k_{o} v}\right]^{5}\left(5+28 t^{2}+24 t^{2}+6 \eta^{2}+8 t^{2} \eta^{2}\right) \\
& \text { where } \\
& t=\tan \varphi f \text { and } \phi f \text { is the footprint latitude, } \\
& \eta^{2}=\frac{e^{2}}{1-e^{2}} \cos ^{2} \varphi, \\
& S=\frac{a}{1+n}\left(a_{0} \varphi-a_{2} \sin 2 \varphi+a_{4} \sin 4 \varphi-\right. \\
& \left.a_{6} \sin 6 \varphi+a_{8} \sin 8 \varphi\right), \\
& a_{0}=1+\frac{n^{2}}{4}+\frac{n^{4}}{64}, a_{2}=\frac{3}{2}\left[n-\frac{n^{3}}{8}\right], \\
& a_{4}=\frac{15}{16}\left[n^{2}-\frac{n^{4}}{4}\right], a_{6}=\frac{315}{512} n^{4}, n=\frac{f}{(2-f)}
\end{aligned}
$$

\subsubsection{Model Adequacy Evaluation}

To assess the transformation strength of the Procrustes approach, horizontal positional accuracy was done by comparing the Procrustes computed coordinates to the known coordinates. Commonly used statistical tools such as Horizontal Error (HE), Root Mean Square Horizontal Error (RMSHE), Standard Deviation (SD) and Arithmetic Mean of the Horizontal Error (AMHE) were used for the performance evaluation. Equations (28) to (31) (Ziggah et al., 2019) present the mathematical notations.

$$
\begin{gathered}
H E=\sqrt{\Delta E^{2}+\Delta N^{2}} \\
R M S H E=\sqrt{\frac{\sum_{i=1}^{n}\left(H E_{i}\right)^{2}}{n}} \\
A M H E=\frac{1}{n} \sum_{i=1}^{n} H E_{i} \\
S D=\sqrt{\frac{1}{n-1} \sum_{i=1}^{n}(H E-\overline{H E})^{2}}
\end{gathered}
$$


where

$\Delta E=E_{\text {known }}-E_{\text {Procrustes }}$ and $\Delta N=N_{\text {known }}-N_{\text {Procrustes }}$.

The $n$ is the total number of points, $\overline{H E}$ is the mean horizontal error value, $(\mathrm{E}, \mathrm{N})_{\text {known }}$ is the known projected Easting and Northing grid coordinates, and $(\mathrm{E}, \mathrm{N})_{\text {Procrustes }}$ is the computed Procrustes coordinates.

\section{Results and Discussion}

The transformation parameters produced by the Procrustes approach are presented in Table 2. The translation parameters indicate the extent at which the WGS84 origin deviate from the War Office 1926 datum along the three-dimensional reference axes. For the rotational parameters, it creates parallelism between the reference axes of the WGS84 and War Office 1926. The scale factor, on the other hand, creates equal dimensions in the references axes of WGS84 and War Office 1926.

The presented coordinate differences $(\Delta \mathrm{E}, \Delta \mathrm{N})$ (Table 3) indicates how much the Procrustes computed coordinates deviated from the known projected grid coordinates. A vivid demonstration on the variability of the residuals with respect to the ideal threshold zero residual value is shown in Fig. 3.

Table 2 Computed Coordinate Transformation Parameters

\begin{tabular}{|c|c|c|c|c|c|}
\hline \multicolumn{2}{|c|}{ Parameters } & \multicolumn{3}{|c|}{ Values } & Units \\
\hline \multirow{2}{*}{\multicolumn{2}{|c|}{ Rotation Matrix }} & 0.999999999999994 & $1.06614146332809 \times 10^{-7}$ & $2.8198636818275 \times 10^{-8}$ & \\
\hline & & $-1.06614207362708 \times 10^{-7}$ & 0.999999999997666 & $2.1581121022736 \times 10^{-6}$ & arc \\
\hline \multirow{3}{*}{ Translation } & $\Delta \mathrm{X}$ & & 151.1909729 & & \multirow{3}{*}{ metre } \\
\hline & $\Delta \mathrm{Y}$ & & -31.59264456 & & \\
\hline & $\Delta \mathrm{Z}$ & & -327.1790029 & & \\
\hline \multicolumn{2}{|l|}{ Scale factor } & & 1.00000716720198 & & $\mathrm{ppm}$ \\
\hline
\end{tabular}

Table 3 Difference between Known and Procrustes Computed Coordinates (Units in metres)

\begin{tabular}{|c|c|c|c|}
\hline Point & $\Delta \mathbf{N}$ & $\Delta \mathbf{E}$ & HE \\
\hline 1 & 1.108 & -0.239 & 1.134 \\
\hline 2 & 0.909 & 0.006 & 0.909 \\
\hline 3 & -1.472 & 0.476 & 1.547 \\
\hline 4 & 1.864 & 0.384 & 1.903 \\
\hline 5 & -0.749 & -0.365 & 0.833 \\
\hline 6 & -0.707 & 0.35 & 0.789 \\
\hline 7 & -0.527 & 1.145 & 1.261 \\
\hline 8 & 0.64 & 0.471 & 0.795 \\
\hline 9 & 0.047 & 0.761 & 0.762 \\
\hline 10 & -0.28 & 0.66 & 0.717 \\
\hline 11 & -0.192 & -0.098 & 0.215 \\
\hline 12 & -0.326 & -0.236 & 0.403 \\
\hline 13 & 1.273 & 0.737 & 1.471 \\
\hline 14 & 1.112 & -0.101 & 1.117 \\
\hline 15 & 0.423 & 1.015 & 1.1 \\
\hline 16 & 0.854 & -0.099 & 0.859 \\
\hline 17 & -0.399 & -0.187 & 0.440 \\
\hline 18 & 0.094 & 0.058 & 0.111 \\
\hline 19 & -0.612 & -0.454 & 0.762 \\
\hline Mean & 0.161 & 0.225 & 0.901 \\
\hline SD & 0.865 & 0.481 & 0.452 \\
\hline & & & \\
\hline
\end{tabular}




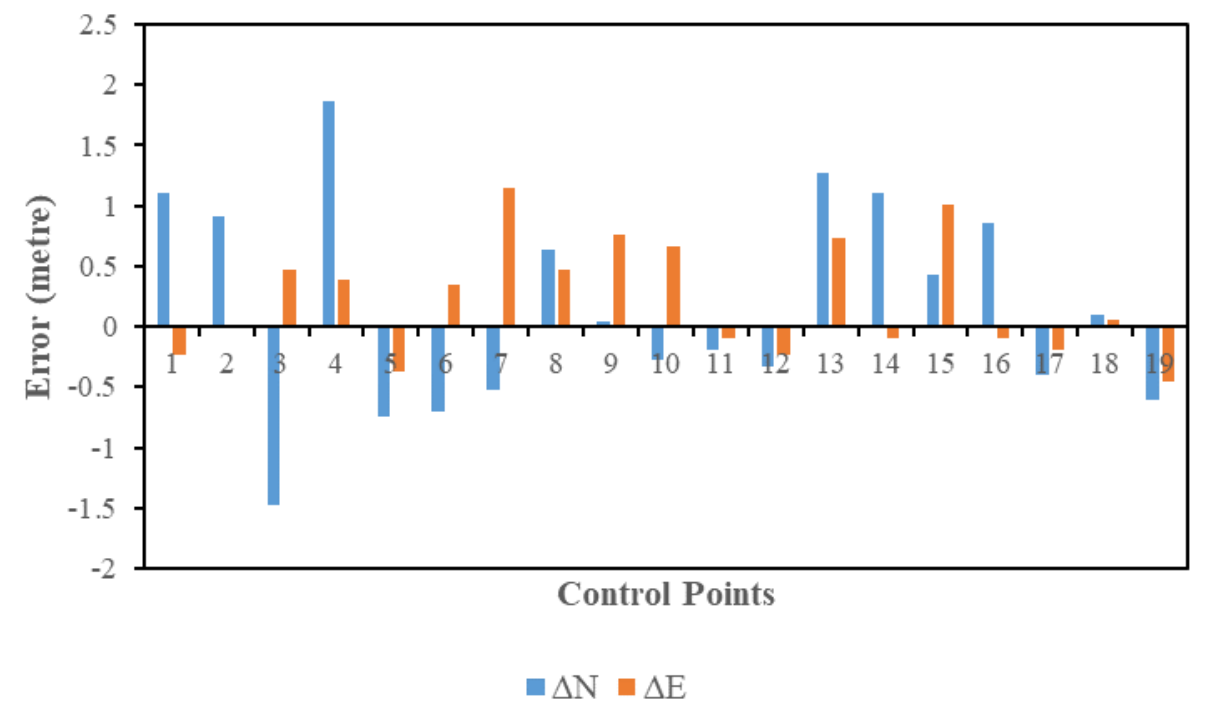

Fig. 3 Variation of Easting and Northing Residuals

The residuals (Table 3) were also plotted (Fig. 4) as velocity vectors with respect to the Procrustes computed projected grid coordinates. The essence is to identify whether the residuals exhibited any systematic or random patterns. In Fig. 4, the direction and length of the arrows show the respective residual values in Easting $(\Delta \mathrm{E})$ and Northing $(\Delta \mathrm{N})$. From the diagram, it can be seen that the residual directions are in a scattershot fashion and appear to be random. Hence, there is no systematic pattern. Inferential analysis of Fig. 4 shows that where there is a wide gap between control points or no existing points, the residual will be large leading to unreliable transformation results for that particular area. However, areas where there is evenly distribution of control points in the study area, better transformation results will be obtained.

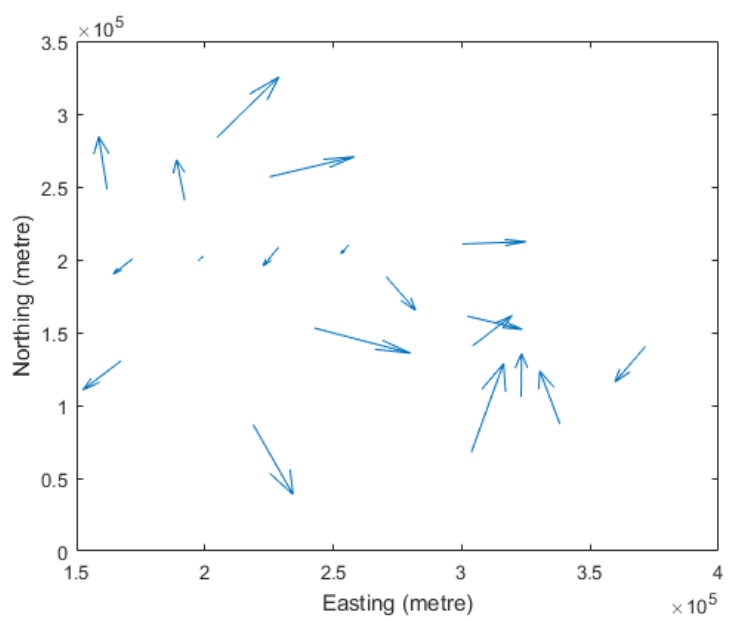

Fig. 4 Residual Vectors of the Procrustes Transformation

In Table 3, the Horizontal Positional Errors (HE) were computed using Equation (28). It is important to note that the HE values describe how closely the Procrustes computed coordinates are compared to the known positional information. It can be observed that the Procrustes approach produced a maximum and a minimum uncertainty values of $1.903 \mathrm{~m}$ and $0.11 \mathrm{~m}$, respectively (Table 4). These values explain quantitatively the horizontal positional error range achieved by the Procrustes approach in the study area. The obtained uncertainties can be attributed to the quality of dataset in the War Office 1926 and the systematic errors usually exhibited in local geodetic networks causing different parts of such networks to have varying distortions in scale and orientations.

Table 4 Summary Statistics for Evaluating the Performance of Procrustes Approach (Units in metres)

\begin{tabular}{|c|c|}
\hline Criteria & Value \\
\hline RMSHE & 1.003 \\
\hline AMHE & 0.901 \\
\hline SD & 0.452 \\
\hline Maximum & 1.903 \\
\hline Minimum & 0.111 \\
\hline
\end{tabular}

To ascertain the accuracy of integrating the data between WGS84 and War Office 1926, the RMSHE was used. In Table 4, a total uncertainty of $1.003 \mathrm{~m}$ was achieved. The Procrustes approach also produced an average horizontal uncertainty of $0.901 \mathrm{~m}$. The standard deviation value $(0.452 \mathrm{~m})$ presents the precision level of the Procrustes computed coordinates with respect to the known coordinates. This means that the transformation results disperse from the most probable value by $0.452 \mathrm{~m}$.

Contrasting to other coordinate transformation results reported in Ghana (Ayer and Fosu, 2008; 
Ziggah et al., 2017, 2019), the transformation accuracy recorded by the Procrustes approach limits its usability to Geographic Information System (GIS), navigation and low-order land surveys in Ghana. The results obtained confirmed the conclusion made in Paláncz et al. (2010) and Awange et al. (2008) that the general Procrustes approach applied in this study sometimes does not perform effectively for networks with mild anisotropy like Ghana geodetic network.

\section{Conclusions and Recommendations}

This study applied and assessed the efficiency of the 3D Procrustean approach to transform coordinates from WGS84 to Ghana War Office 1926. The Procrustes approach produced a transformation precision of $0.452 \mathrm{~m}$ based on the standard deviation. A root mean square horizontal error $(1.003 \mathrm{~m})$ and average horizontal error $(0.901$ $\mathrm{m})$ values were also achieved. The practicality of these results limit the 3D Procrustean approach application to the area of Geographic Information System (GIS), Navigation and any low-order land surveys in Ghana. The Procrustes approach limitation of not able to produce high surveyaccuracy coordinate values in Ghana geodetic reference network may be due to the network distortions and quality of data associated with it.

For future studies, the focus will be on applying the Awange-Bae-Claessens's solution (ABC method) and Paláncz-Zaletnyik's solution (PZ method) that have been extended and generalized to the $3 \mathrm{D}$ affine transformation. These methods have been noted to perform well in mild anisotropy networks than applying the general Procrustes to the Helmert transformation.

\section{Acknowledgement}

The authors acknowledge the use of data from the Land Administration Project (LAP) of the Ghana Survey and Mapping Division of Lands Commission.

\section{References}

Awange, J. L. and Grafarend, E. W. (2005), Solving Algebraic Computational Problems in Geodesy and Geoinformatics, Springer Berlin, Germany, 333 pp.

Awange, J.L. and Grafarend, E.W. (2003), “Closed form solution of the over determined nonlinear 7 parameter datum transformation", Allgemeine Vermessungsnachrichten, Vol. 110, pp. 130149.
Awange, J.L. and Paláncz, B. (2016), Geospatial algebraic computations: Theory and applications, 3rd Edition, Springer Berlin, 541 pp.

Awange, J.L., Bae, K.H. and Claessens, S.J. (2008), Procrustean solution of the 9-parameter transformation problem, Earth, Planets and Space, Vol. 60, No. 6, pp. 529-537.

Ayer, J. (2008), "Transformation Models and Procedures for Framework Integration of the Ghana National Geodetic Network", The Ghana Surveyor, Vol. 1, No. 2, pp. 52-58.

Ayer, J. and Fosu, C. (2008), "Map coordinates referencing and the use of GPS datasets in Ghana", Journal of Science and Technology, Vol. 28, No. 1, pp. 116-127.

Bowring, B.R. (1976), "Transformation from spatial to geodetic coordinates" Survey Review, Vol. 23, No. 181, pp. 323-327.

Dzidefo, A. (2011), "Determination of Transformation Parameters between the World Geodetic System 1984 and the Ghana Geodetic Network", Master's Thesis, Department of Civil and Geomatic Engineering, KNUST, Kumasi, Ghana, pp. 1-97.

Fosu, C., Poku-Gyamfi, Y. and Hein, W.G. (2006), "Global Navigation Satellite System (GNSS) A Utility for Sustainable Development in Africa", 5th FIG Regional Conference on Promoting Land Administration and Good Governance, Workshop - AFREF I, Accra, Ghana, pp. 1-12.

Gower, J.C. and Dijksterhuis, G.B. (2004), Procrustes problems, Oxford Statistical Series Vol. 30, Oxford University Press on Demand, $248 \mathrm{pp}$.

Grafarend, E.W., and Awange, J.L. (2003), "Nonlinear analysis of the three-dimensional datum transformation [conformal group C7 (3)]", Journal of Geodesy, Vol. 77, pp. 66-76.

Hofmann-Wellenhof, B. and Moritz, H. (2006), Physical geodesy. 2nd Edition, Springer Science \& Business Media, Austria, 402 pp.

Kotzev, V. (2013), "Consultancy Service for the Selection of a New Projection System for Ghana." Unpublished Draft Final Reports, World Bank Second Land Administration Project (LAP-2), Ghana, (Unpublished), 16 pp.

Mugnier, J.C. (2000), OGP-coordinate conversions and transformations including formulae. COLUMN, Grids and Datums. The Republic of Ghana. Photogrammetric Engineering and Remote Sensing, pp. 695-697.

Paláncz, B., Zaletnyik, P., Awange, J.L. and Heck, B. (2010), Extension of the ABC-Procrustes algorithm for 3D affine coordinate transformation. Earth, Planets and Space, Vol. 62, No. 11, pp. 857-862.

Păun, C.D., Oniga, V.E. and Dragomir, P.I. (2017), "Three-dimensional transformation of coordinate systems using nonlinear analysis- 
Procrustes algorithm", International Journal of Engineering Sciences \& Research Technology, Vol. 6, No. 5, pp. 355-363.

Poku-Gyamfi, Y. (2009). "Establishment of GPS Reference Network in Ghana." PhD Dissertation, Universitat der Bundeswehr Munchen, Germany, pp. 1-177.

Poku-Gyamfi, Y. and Schueler, T. (2008), "Renewal of Ghana's geodetic reference network", In 13th FIG Symposium on Deformation Measurement and Analysis, 4th IAG Symposium on Geodesy for Geotechnical and Structural Engineering, LNEC, LISBON, pp. 1-9.

Wonnacott, R. (2007), “A Progress Report on The AFREF Project and its potential to support development in Africa", Space Geodesy Workshop, Matjiesfontein, 13-14 November, pp. 1-29.

Ziggah, Y. Y., Youjian, H., Tierra, A. R. and Laari, P. B. (2019), "Coordinate transformation between global and local data based on artificial neural network with K-fold cross-validation in Ghana", Earth Sciences Research Journal, Vol. 23, No. 1, pp. 67-77.

Ziggah, Y.Y., Youjian, H., Laari, P.B. and Hui, Z. (2017), "Novel approach to improve geocentric translation model performance using artificial neural network technology", Boletim de Ciências Geodésicas, Vol.23, No.1, pp.213-23.

\section{Authors}

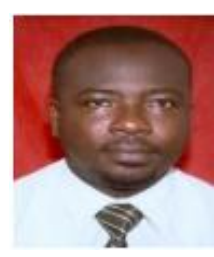

B. Kumi-Boateng is an Associate Professor at the Department of Geomatic Engineering of the University of Mines and Technology (UMaT), Tarkwa, Ghana. He holds a Bachelor of Science degree in Geomatic Engineering from the Kwame Nkrumah University of Science and Technology, Kumasi, Ghana. He obtained his Master of Science degree and Doctor of Philosophy from the International Institute for Geo-information Science and Earth Observation (ITC), Enschede-The Netherlands and UMaT respectively. His research interest includes application of Remote Sensing and GIS in Environmental Management, Spatial Statistics, Land and Compensation Surveys as well as engineering application of artificial intelligence, geodetic coordinate transformation, gravity field modelling, height systems and geodetic deformation modelling.

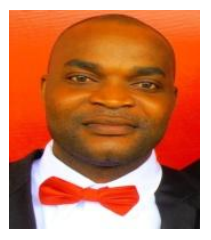

Y. Yevenyo Ziggah is a Lecturer at the Geomatic Engineering Department of the University of Mines and Technology (UMaT). He holds a BSc in Geomatic Engineering from Kwame Nkrumah University of Science and Technology, Kumasi, Ghana. He obtained his Master of Engineering degree and Doctor of Philosophy in Geodesy and Survey Engineering from China University of Geosciences (Wuhan). His research interests include engineering application of artificial intelligence, geodetic coordinate transformation, gravity field modelling, height systems and geodetic deformation modelling. 